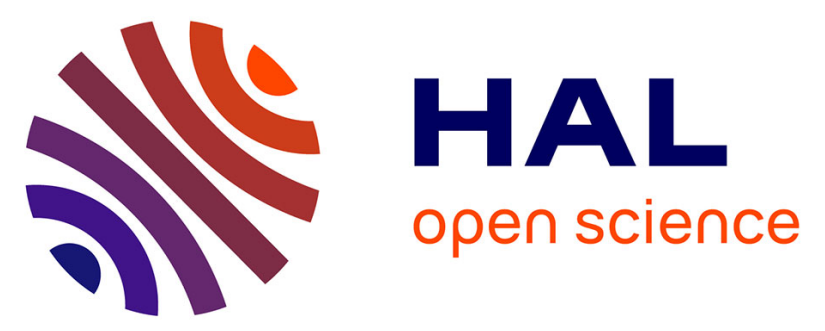

\title{
Relationship between change in physical activity and in clinical status in patients with idiopathic inflammatory myopathy: A prospective cohort study
}

Océane Landon-Cardinal, Damien Bachasson, Perrine Guillaume-Jugnot, Mathieu Vautier, Nicolas Champtiaux, Baptiste Hervier, Aude Rigolet, Rohit Aggarwal, Olivier Benveniste, Jean-Yves Hogrel, et al.

\section{To cite this version:}

Océane Landon-Cardinal, Damien Bachasson, Perrine Guillaume-Jugnot, Mathieu Vautier, Nicolas Champtiaux, et al.. Relationship between change in physical activity and in clinical status in patients with idiopathic inflammatory myopathy: A prospective cohort study. Seminars in Arthritis and Rheumatism, 2020, 50, pp.1140 - 1149. 10.1016/j.semarthrit.2020.06.014 . hal-03491516

\author{
HAL Id: hal-03491516 \\ https://hal.science/hal-03491516
}

Submitted on 12 Jan 2022

HAL is a multi-disciplinary open access archive for the deposit and dissemination of scientific research documents, whether they are published or not. The documents may come from teaching and research institutions in France or abroad, or from public or private research centers.
L'archive ouverte pluridisciplinaire HAL, est destinée au dépôt et à la diffusion de documents scientifiques de niveau recherche, publiés ou non, émanant des établissements d'enseignement et de recherche français ou étrangers, des laboratoires publics ou privés. 


\title{
Relationship between change in physical activity and in clinical status in patients with idiopathic inflammatory myopathy: A prospective cohort study
}

\author{
Océane Landon-Cardinal ${ }^{\mathrm{a}, \mathrm{b}, 1, *}$, Damien Bachasson ${ }^{\mathrm{c}, 1}$, Perrine Guillaume-Jugnot ${ }^{\mathrm{a}}$, \\ Mathieu Vautier ${ }^{\mathrm{a}}$, Nicolas Champtiaux ${ }^{\mathrm{a}}$, Baptiste Hervier ${ }^{\mathrm{a}}$, Aude Rigolet ${ }^{\mathrm{a}}$, Rohit Aggarwal $^{\mathrm{d}}$, \\ Olivier Benveniste ${ }^{a, c}$, Jean-Yves Hogrel ${ }^{c}$, Yves Allenbach ${ }^{a, c}$
}

\footnotetext{
a Department of Internal Medicine and Clinical Immunology and Inflammation-Immunopathology-Biotherapy Department (I2B), Pitié-Salpêtrière University Hospital, Assistance Publique-Hôpitaux de Paris, East Paris Neuromuscular Diseases Reference Center, Inserm U974, Sorbonne Université, Paris 6, Paris, France

${ }^{\mathrm{b}}$ Department of Medicine, University of Montreal; Division of Rheumatology and Research Center, Centre Hospitalier de l'Université de Montréal, Montreal, QC, Abbreviation

${ }^{\mathrm{c}}$ Institute of Myology, Neuromuscular Investigation Center, Pitié-Salpêtrière University Hospital, Paris, France

${ }^{\mathrm{d}}$ Division of Rheumatology and Clinical Immunology, Department of Medicine, University of Pittsburgh, Pittsburgh, Palestine, State of, United States
}

\section{A R T I C L E I N F O}

\section{Keywords:}

Idiopathic inflammatory myopathy

Myositis

Autoimmune myopathy

Physical activity

Accelerometer

Outcome measure

\begin{abstract}
A B S T R A C T
Objective: This study aimed to investigate the relationship between changes in clinical status on daily life physical activity (PA) in patients with idiopathic inflammatory myopathy (IIM).

Methods: Patients with dermatomyositis (DM), immune-mediated necrotizing myopathy (IMNM) or overlap myositis (OM) who presented either a new-onset or relapsing IIM, stable disease on maintenance therapy or were undergoing immunosuppressant tapering were included. Patients were evaluated at inclusion (V0), and at two follow-up visits (V1, 94 \pm 12 days from V0; V2, 96 \pm 17 days from V1). The American College of Rheumatology/European League against Rheumatism (ACR/EULAR) response criteria was recorded. PA assessed using 14-days raw accelerometry data gathered using a wrist-worn accelerometer after each visit (mean daily Euclidean norm minus $1 \mathrm{~g}$ (ENMO) was computed).

Results: Fifty-five patients (16 OM, 27 IMNM and 12 DM) were included. At baseline, 67\% of patients had an ENMO Z-score less than 1. At inclusion, ENMO mainly correlated with health assessment questionnaire score (HAQ $\rho=-0.51, p<0.01$ ), manual muscle testing 8 (MMT8, $\rho=0.42, p<0.01$ ), creatinine level $(\rho=0.41, p<0.01$ ), and SF-36 physical functioning score $(\rho=0.38, p<0.002)$. At follow-up, ENMO changes mainly correlated with changes in MMT8, HAQ, SF-36 fatigue, and depression score (all $\rho>0.43$, all $p<0.001$ ). Level of agreement between ACR/EULAR response criteria and changes in PA was 15, 45, and 90\% for minimal $(n=13)$, moderate $(n=20)$, and major $(n=10)$ improvements, respectively.

Conclusion: Baseline PA levels and change in PA correlated with muscle strength and function, yet changes in PA were also influenced by psychological status. Only patients with major improvements on the ACR/EULAR criteria had significant increase in PA. Accelerometer may serve as an objective tool to define a clinically relevant real-life outcome.
\end{abstract}

(c) 2020 Elsevier Inc. All rights reserved.

\section{Introduction}

Idiopathic inflammatory myopathies (IIM) are a very heterogeneous group of autoimmune muscle diseases impairing patients' quality of life [1]. They can be classified into four main subsets: dermatomyositis (DM), immune-mediated necrotizing myopathy (IMNM), overlap

\footnotetext{
* Corresponding author. Department of Internal Medicine and Clinical Immunology, Pitié-Salpêtrière University Hospital, 47-83 Boulevard de l'Hôpital, 75013 Paris, France

E-mail address: o.landoncardinal@gmail.com (O. Landon-Cardinal).

1 These authors contributed equally to this work
}

myositis (OM) and inclusion body myositis (IBM) [2]. Heterogeneity within each subset is related to the variability of muscle involvement (weakness severity, dysphagia, heart or respiratory muscle involvement) and extra-muscular features. Thus, IIM assessment in clinical practice and in the design of clinical trials remains challenging. Improving management requires reliable tools to assess disease activity and evaluate the impact of IIM impact on quality of life. Novel tools are crucial not only for improving patients' management in clinical practice but also for assessing new therapeutic options in clinical trials.

The International Myositis Assessment \& Clinical Studies Group (IMACS) developed a core-set measure (CSM) to capture both 
muscular and/or extra-muscular disease activity in IIM [3]. The CSM includes: a manual muscle testing (MMT), muscle enzymes, Health Assessment Questionnaire (HAQ), a physician global disease activity, a patient global disease activity, and one item is specifically developed to assess the extra-muscular involvement (extra-muscular global disease activity). Using these CSM, the American College of Rheumatology/European League against Rheumatism (ACR/EULAR) recently developed criteria for clinical response in IIM based on the absolute percent change of the CSM between two time-points [4].

Yet, clinical meaningfulness of this clinical response (mild, moderate and major) in patients' daily life remains to be determined. Muscle weakness may alter functional abilities of patients with IIM and therefore impact their daily physical activity (PA). Moreover, daily PA may also be affected by the cardiorespiratory state, pain level, extramuscular features, as well as psychological and behavioral components. Analysis of raw multiday wrist-worn triaxial accelerometers data using open-source tools $[5,6]$ has been identified as an efficient way to objectively quantify PA. We used this approach in a recent pilot study and highlighted that clinical changes were associated with changes in PA in patients with IIM $[7,8]$. However, the complex interplay between changes in clinical/biological outcomes and changes in real-life PA remains unknown.

The aim of this study was therefore to investigate relationships between PA levels, changes in PA levels, and IMACS CSM and ACR/ EULAR response criteria in patients with IIM.

\section{Materials and Methods}

\section{Study design}

A prospective single center study was performed at our national adult referral center for neuromuscular diseases. All patients were adults and were evaluated as part of routine clinical care at the outpatient clinic or during hospitalization at inclusion (V0, from February 2017 through April 2018). Patients were re-evaluated during two follow-up visits, (V1, $94 \pm 12$ days from V0; V2, 96 \pm 17 days from V1).

\section{Participants}

Included patients were classified with an IIM according to the 2017 EULAR/ACR classification criteria [9] and were further divided into four subgroups: DM, IMNM (European NeuroMuscular Center criteria) [10], or an OM, including anti-synthetase syndrome [11,12]. Patients with IBM [13] were not enrolled, since standard immunosuppressive therapy has not been shown to improve the disease course [14] and the ACR/EULAR clinical response criteria was not designed for this IIM subgroup. Patients were enrolled at disease onset, during a relapse, or those with stable disease, either on maintenance therapy or while undergoing immunosuppressant tapering.

Myositis-specific and myositis-associated antibodies testing was recorded at the time of myositis diagnosis and was performed using line blot commercial assay (EUROIMMUN, Lübeck, Germany) and included: anti-SAE, -TIF1 $\gamma$, -NXP2, -MDA5, -Mi-2, -Jo1, -PL-12, -PL-7, -SRP, -OJ, -EJ. Anti-HMGCR antibodies were identified using ELISA (Inova Diagnostics Inc.) or addressable laser bead immunoassay (ALBIA).

\section{Muscle disease assessment}

\section{Muscle tests}

Muscle strength was assessed using the MMT-8 (8 muscle groups, 10-point Kendall scale) [15]. MMT of the psoas was performed but was not used to compute the MMT-8 total score. Quantitative muscle strength of the deltoid (i.e. shoulder abduction) and psoas (i.e. hip flexion) was performed using a hand-held dynamometer (HHD) (MicroFet2 $^{\mathrm{TM}}$; Hoggan Health Industries, USA) following standardized procedures [16] and expressed as absolute values and as percentages of predicted values, using previously published equations [17].

Additionally, endurance assessments were performed in the upper limbs and the lowers limbs as previously reported [18]:

- Barré test: time in seconds ( $\mathrm{sec}$ ) during which a patient can maintain his arms in flexion at a $90^{\circ}$ angle with completely extended elbows. The value is set to 240 if the duration exceeds $240 \mathrm{~s}$.

- Mingazzini test: time in second during which a patient can maintain the legs elevated with the hips and the knees bent at a $90^{\circ}$ angle, in the supine position. The value is set to 100 if the duration exceeds $100 \mathrm{~s}$.

All muscle assessments were performed by the same trained clinician (OLC). Physician and patient global disease activity using a 10$\mathrm{cm}$ visual analog scale (VAS), serum creatine kinase (CK, normal range $=22-198 \mathrm{IU} / \mathrm{L}$ ), aspartate aminotransferase (AST, normal range $=6-34 \mathrm{IU} / \mathrm{L}$ ), alanine aminotransferase (ALT, normal range $=7-55 \mathrm{IU} / \mathrm{L}$ ), lactate dehydrogenase (LDH, normal range $=140-280 \mathrm{IU} / \mathrm{L}$ ) and creatinine levels (normal range $=62-106$ $\mu \mathrm{mol} / \mathrm{L}$ ), and HAQ were also assessed at each visit.

\section{Physical activity assessment}

Physical activity monitoring and data collection. PA was assessed using a widely-used wrist-worn accelerometer (GENEActiv ${ }^{\circledR}$, Activinsights Ltd, Kimbolton, UK), which was worn on the non-dominant wrist of patients at all time (e.g. day, night, shower) for 14 consecutive days after each visit. This duration has been demonstrated as a comfortable time window to capture daily PA $[19,20]$.

The GENEActiv accelerometers measure triaxial movement acceleration in gravity units $\left(1 \mathrm{~g}=9.81 \mathrm{~m} / \mathrm{s}^{2}\right)$ at a frequency of $50 \mathrm{~Hz}$. Participants continued their daily life and no instructions were provided to patients regarding both structured and spontaneous PA. At each patients' visit, participants were asked to estimate (i.e. number and duration of sessions per week then analyzed as minutes per week) any structured PA (e.g. exercise training, gymnastics, swimming) and/or physical therapy sessions they have been performing within 2 weeks prior to each visit.

Processing of raw accelerometer data. Following the measurement period, raw accelerometer data were extracted using the manufacturer software and processed using the open-source GGIR Rpackage [https://cran.r-project.org/web/packages/GGIR] [5,6]. To ensure different devices provided a similar output under similar conditions, the acceleration signals to local gravity were calibrated using the procedure described by van Hees et al. [5]. The Euclidean distance is the straight-line distance between two points in Euclidean space forming a metric space and the associated norm is called the Euclidian norm measuring the length of the vector. The vector magnitude from the three axes corrected for gravity referred to as Euclidean norm minus $1 \mathrm{~g}$ (gravitational constant) (ENMO) was calculated [5]. This procedure allows for processing the raw acceleration data and attempting to separate the movement and gravitational components of the signal [6]. ENMO is presented in milli-g ( $\mathrm{mg}$ with $1 \mathrm{mg}=0.00981 \mathrm{~m} / \mathrm{s}^{2}$ ) per day. The procedure for non-wear detection was performed as previously described [6]. Minimal worn time per days for day-specific analyses was set at $16 \mathrm{~h}$. Duration of inactivity, light PA, and moderate to vigorous PA were computed using previously validated thresholds [21]: inactivity $<30 \mathrm{mg} ; 30 \mathrm{mg} \leq$ light $\mathrm{PA}<100 \mathrm{mg} ; 100 \mathrm{mg} \leq$ moderate $\mathrm{PA}<400 \mathrm{mg}$; vigorous $\mathrm{PA} \geq$ $400 \mathrm{mg}$. Moderate to vigorous activity accumulated in $\geq 10 \mathrm{~min}$ bouts for which $80 \%$ of the epochs are above $100 \mathrm{mg}$ was also calculated. ENMO Z-scores accounting for age and sex were computed based on accelerometer measured PA in over 100,000 participants within the 
UK Biobank study [22]. ENMO Z-scores were used to assess PA levels in IIM patients at inclusion compared to healthy population.

Extra-muscular disease assessment

The extra-muscular disease activity VAS, a quality of life questionnaire (SF-36 version 2) [23] and the Hospital Anxiety and Depression scale (HAD) [24] were completed at each visit.

\section{ACR/EULAR IIM response criteria}

As per the ACR/EULAR response criteria, a total score change of $<20, \geq 20, \geq 40$ or $\geq 60$ was considered as an unchanged, minimal, moderate or major improvement, respectively [4]. In addition, improvement scores were calculated for each CSM [4] as well as absolute percent changes for all recorded variables. For the purpose of statistical analysis only, patients with CSM negative CSM scores were also taken into account to compute worsening clinical status. An adapted ACR/EULAR worsening criteria was defined as follows: $-40 \leq$ minimal worsening $\leq-20 ;-60 \leq$ moderate worsening $\leq$ $-40 ; \geq-60$ major worsening.

\section{Statistics}

Quantitative variables are reported as mean \pm standard deviation or mean (95\% confidence interval [lower 95\% CI, upper 95\% CI]). Oneway ANOVAs were conducted to investigate difference between IIM subgroups at inclusion in normally distributed variables. Bonferroni's post-hoc tests were performed if ANOVAs demonstrated a significant main effect of subgroups on variables. Repeated measure ANOVAs were used to investigate changes at follow-up with Bonferroni's post-hoc tests in normally distributed variables. Friedman test with Bonferroni's post-hoc tests were used in non-normally distributed variables. Cohen's d effect sizes were also computed to compare differences between subgroups (e.g. as defined by the ACR/EULAR response criteria). To assess inter-week reliability of ENMO, change in mean and paired t-tests were used for detection of systematic bias. Standard error of measurement was used to study absolute reliability. Relative reliability was assessed with the intra-class correlation coefficient with $95 \%$ confidence interval. Regression analysis and Bland-Altman plots were also performed. Least significant change for ENMO was defined as twice the standard error of measurement between mean ENMO of the first and second week from all available recordings. Least significant change for ENMO was used to classify changes in PA. Categorical variables were compared using a $\chi^{2}$. Kappa statistic was used to test for agreement of the adapted ACR/EULAR status and changes in PA (for this analysis, the adapted ACR/EULAR status was simplified to impaired, unchanged, improved) Potential relationships between variables were investigated using Spearman correlation coefficient. For investigating relationship between changes in PA and changes in other variables, changes between V0 and follow-up visits (V1, V2) were analyzed jointly. Random forest regression analysis was also used to identify most important variable influencing PA at baseline and changes in PA at follow-up. For all statistical analyses, $p<0.05$ was considered significant. All statistical analyses were performed in R 3.4.3 Team RC. R: A Language and Environment for Statistical Computing. 2017.

\section{Ethics statement}

The study conformed to the Declaration of Helsinki and was approved by local Ethics Committee (CPP Ile-De France VI (2013-12-19), CCTIRS ( $\left.{ }^{\circ} 14.323\right)$ and CNIL $\left.(915,139)\right)$ for the use of medical information recorded in the IIM database for scientific purposes. All participants gave written informed consent.

\section{Results}

\section{Participants}

Fifty-nine patients were enrolled, and four patients were excluded either because they were lost to follow-up $(n=3)$ or because they failed to perform $\geq 2 / 3$ accelerometer recordings $(n=1)$. The characteristics of the 55 included patients (12 DM, 27 IMNM and $16 \mathrm{OM}$ ) are presented in Table 1. Myositis-specific antibodies were anti-TIF1gamma $(n=4)$, -NXP-2 $(n=3)$, -Mi-2 $(n=1),-\mathrm{SAE}(n=1)$ in DM; antiHMGCR $(n=18)$, -SRP $(n=7)$ in IMNM; and anti-Jo1 $(n=4)$, -PL7 $(n=1),-\mathrm{EJ}(n=1)$ in OM. Most patients were treated with a combination of corticosteroids and immunosuppressants, with or without intravenous immunoglobulin. Three patients had a combination of rituximab with either methotrexate $(n=2)$ or mycophenolate mofetil $(n=1)$.

Patients with IMNM presented a longer disease duration and lower extra-muscular global involvement compared to the other subsets and a poorer SF-36 social functioning, physical role, emotional role and emotional wellbeing compared to patients with DM (Table 1). On average, baseline quantitative muscle strength, expressed as a percentage of predicted strength, was reduced in psoas and to a lesser extent in deltoids. Consistently, endurance (Barré and Mingazzini tests) measured at baseline was largely reduced in the lower limbs and was less importantly affected in the upper limbs. Variable values and changes at inclusion and follow-up visits are displayed in Table 2.

Assessing physical activity with wrist-worn accelerometer is feasible and reliable in patients with IIM

Wear-time compliance of triaxial wrist-worn accelerometers was $13.7 \pm 1.1$ days. There was no change in wear-time compliance between time points $(p=0.87)$. Number of valid week days was $9.8 \pm 0.8$ (maximum of 10 days) and number of valid weekend days was $3.9 \pm 0.3$ (maximum of 4 days). Of note, no calibration error (i.e. estimated based on all 'non-movement' periods in the measurement after applying the autocalibration) was reported. Non-wear time expressed as a percentage of total measurement duration was $0.12 \pm$ $0.53 \%$. Standard error of measurement expressed as a percentage was $10.01[8.97,11.32] \%$ and intraclass correlation coefficient $>0.9$. Interweek reliability of ENMO is displayed in Fig. 1A. ENMO least significant change was $4.10 \mathrm{mg} /$ day.

\section{Low physical activity in most patients with IIM compared to the general population}

PA metrics at baseline are shown in Fig. 1. Fifty-four percent of patients had a Z-score between -1 and -2 and $12 \%$ had Z-score $<-2$. There was no significant difference in PA metrics across disease subgroups (all $p>0.16$ ). PA intensity was similar in all subsets and was largely dominated by light PA (average $193 \pm 74 \mathrm{~min} /$ day), while moderate to vigorous PA was only performed on average performed only $6 \pm 15 \mathrm{~min} /$ day. Average duration of physical therapy sessions and self-structured PA was $33 \pm 66$ and $14 \pm 40 \mathrm{~min} /$ week, respectively. There was no correlation between ENMO and physical therapy or structured PA (both $p>0.19$ ).

\section{At inclusion, physical activity correlates with muscle strength and patients reported outcome of physical function}

Relationships between PA and variables at inclusion (V0) are shown in Table 3. Variables significantly correlated with ENMO Zscore at V0 are displayed in Fig. 2. At V0, ENMO mainly correlated with the HAQ MMT8, creatinine levels, and SF-36-physical functioning. Interestingly, the other variables included in the CSM were not correlated with ENMO (physician-reported global disease activity, 
Table 1

Patient characteristics at baseline.

\begin{tabular}{|c|c|c|c|c|}
\hline & Variables & $\mathrm{DM}(n=12)$ & $\operatorname{IMNM}(n=27)$ & $\mathrm{OM}(n=16)$ \\
\hline \multirow[t]{5}{*}{ Demographics } & Age (yr) & $48 \pm 17$ & $54 \pm 15$ & $49 \pm 15$ \\
\hline & Disease duration (yr) & $3 \pm 2^{\#}$ & $9 \pm 8^{*}$ & $3 \pm 4$ \\
\hline & Female (\%) & 83 & 63 & 88 \\
\hline & Time from diagnosis (yr) & $2.3 \pm 2$ & $4.2 \pm 4.2^{*}$ & $0.7 \pm 1.4$ \\
\hline & Time from treatment initiation (yr) & $2.1 \pm 2.3$ & $4.0 \pm 4.2^{*}$ & $0.6 \pm 1.4$ \\
\hline \multirow[t]{5}{*}{ Laboratory } & MAA (\%) & 0 & 0 & 19 \\
\hline & MSA (\%) & 75 & 93 & 50 \\
\hline & CK level (IU/L) & $620 \pm 927$ & $1182 \pm 3521$ & $4292 \pm 9531$ \\
\hline & AST level (IU/L) & 77 & 50 & 118 \\
\hline & ALT level (IU/L) & 56 & 50 & 102 \\
\hline \multirow[t]{7}{*}{ Treatment } & Prednisone (\%) & 75 & 67 & 88 \\
\hline & Prednisone (mg/day) & $25 \pm 23$ & $12 \pm 14$ & $35 \pm 28$ \\
\hline & Methotrexate (\%) & $0^{\#}$ & 41 & 25 \\
\hline & Azathioprine (\%) & 16 & 11 & 0 \\
\hline & Mycophenolate mofetil (\%) & 25 & 15 & 6 \\
\hline & Rituximab (\%) & 17 & 19 & 0 \\
\hline & $\operatorname{IVIg}(\%)$ & 50 & 52 & 44 \\
\hline \multirow[t]{11}{*}{ Muscle assessment } & MMT8 total score $(0-150)$ & $137 \pm 16$ & $133 \pm 26$ & $139 \pm 9$ \\
\hline & MMT8 left deltoid & $9 \pm 1$ & $8 \pm 3$ & $8 \pm 1$ \\
\hline & MMT8 right deltoid & $9 \pm 1$ & $8 \pm 3$ & $9 \pm 1$ \\
\hline & MMT8 left psoas & $8 \pm 3$ & $6 \pm 4$ & $8 \pm 2$ \\
\hline & MMT8 right psoas & $8 \pm 3$ & $6 \pm 4$ & $8 \pm 2$ \\
\hline & Left Deltoid HHD (\% pred) & $99 \pm 24$ & $92 \pm 29$ & $98 \pm 23$ \\
\hline & Right Deltoid HHD (\% pred) & $96 \pm 22$ & $92 \pm 25$ & $101 \pm 30$ \\
\hline & Left Psoas HHD (\% pred) & $54 \pm 11$ & $50 \pm 20$ & $62 \pm 18$ \\
\hline & Right Psoas HHD (\% pred) & $60 \pm 15$ & $53 \pm 18$ & $64 \pm 18$ \\
\hline & Barré $(0-150 \mathrm{~s})$ & $94 \pm 50$ & $117 \pm 56$ & $130 \pm 42$ \\
\hline & Mingazzini $(0-75 \mathrm{~s})$ & $47 \pm 26$ & $35 \pm 33$ & $44 \pm 31$ \\
\hline \multirow[t]{3}{*}{ Global assessment } & Extra-muscular global activity $(0-10)$ & $2 \pm 1^{\#}$ & $0 \pm 0^{*}$ & $2 \pm 2$ \\
\hline & Physician global activity $(0-10)$ & $3 \pm 2$ & $2 \pm 2$ & $3 \pm 3$ \\
\hline & Patient global activity $(0-10)$ & $5 \pm 2$ & $4 \pm 3$ & $5 \pm 12$ \\
\hline \multirow[t]{13}{*}{ Patient-reported outcome } & $\mathrm{HAQ}$ & $1.4 \pm 0.7$ & $1.1 \pm 0.9$ & $1.3 \pm 0.7$ \\
\hline & HAD-anxiety & $9 \pm 5$ & $7.5 \pm 3$ & $8.3 \pm 4.4$ \\
\hline & HAD-depression & $4 \pm 3$ & $5 \pm 4$ & $7 \pm 4$ \\
\hline & SF-36-general health (\%) & $41 \pm 23$ & $45 \pm 21$ & $42 \pm 20$ \\
\hline & SF-36-physical functioning (\%) & $41 \pm 23$ & $55 \pm 33$ & $44 \pm 27$ \\
\hline & SF-36-social functioning (\%) & $40 \pm 27^{\#}$ & $66 \pm 31$ & $57 \pm 30$ \\
\hline & SF-36- physical role (\%) & $50 \pm 25$ & $60 \pm 27$ & $48 \pm 25$ \\
\hline & SF-36- emotional role (\%) & $42 \pm 30$ & $65 \pm 32$ & $54 \pm 31$ \\
\hline & SF-36-emotional wellbeing (\%) & $51 \pm 14$ & $62 \pm 17$ & $54 \pm 24$ \\
\hline & SF-36-energy/fatigue (\%) & $37 \pm 21$ & $47 \pm 16$ & $39 \pm 24$ \\
\hline & SF-36-pain (\%) & $55 \pm 24$ & $71 \pm 24$ & $63 \pm 28$ \\
\hline & Physical therapy (min/week) & $31 \pm 71$ & $48 \pm 79$ & $11 \pm 25$ \\
\hline & Structured physical activity (min/week) & $20 \pm 52$ & $18 \pm 44$ & $5 \pm 19$ \\
\hline
\end{tabular}

DM, dermatomyositis; IMNM, immune mediated necrotizing myopathy; OM, overlap myositis; Yr, year; MAA myositis-associated antibodies; MSA, myositis-specific antibodies; CK, creatine kinase; IVIg, intravenous immunoglobulin; MMT8, manual muscle testing 8; HHD, hand held dynamometry; HAQ Health Assessment Questionnaire; HAD, Hospital Anxiety and Depression scale; SF-36, Short Form Health Survey; Bonferroni's post-hoc tests were performed if ANOVA demonstrated significant main effect of subgroups on normally distributed variables; Dunn's post-hoc tests were performed if Kruskal-Wallis test demonstrated significant main effect of subgroups in non-normally distributed variables * significant difference between IMNM and $\mathrm{OM}$

\# significant difference between IMNM and DM, \$ significant difference between DM and OM. Except for SF-36 variables,\% indicates proportion/prevalence.

patient-reported global disease activity, muscle enzymes and extramuscular disease activity).

According to random forest regression, most important features associated with ENMO Z-scores were also the HAQ MMT8, creatinine levels, and SF-36-physical functioning (i.e. relative predictor importance was $1.0,0.66,0.66$, and 0.80 , respectively).

At follow-up, changes in physical activity metrics correlates with changes in muscle strength, patients reported outcome of physical function and depression score

Values and changes in variables at follow-up are displayed in Table 2. Relationships between PA and variables at follow-up are shown in Table 3. Variables significantly correlated to absolute changes in ENMO at follow-up are displayed in Fig. 3. At follow-up, changes in ENMO mainly correlated with ACR/EULAR response criteria, changes in HAD-depression, HAQ SF-36 fatigue, and MMT8.
Interestingly, all the variables included in the CSM were significantly correlated with changes in ENMO.

According to random forest regression, the most important features associated with changes in ENMO were absolute percent changes in physician global disease activity, CK/ALT/AST levels, MMT8, SF-36-energy/fatigue, HAQ SF-36-physical functioning, and HAD-depression (i.e. relative predictor importance was 1.0, 0.99, $0.95,0.85,0.95,0.85,0.83,0.78$, and 0.76 , respectively).

Only major improvement of the ACR/EULAR response criteria is strongly associated with significant increase in physical activity

Using the least significant change in ENMO ( $>4.10 \mathrm{mg} /$ day $)$ at follow-up, we observed that: $8.8 \%$ of patients had lower PA, $66.6 \%$ of patients had unchanged PA, and $26 \%$ of patients had increased PA. Using our adapted ACR/EULAR response criteria, $2.2 \%$ of patients had moderate worsening, $2.2 \%$ of patients had minimal worsening, 
Table 2

Values and changes in variables at inclusion and follow-up visits.

\begin{tabular}{|c|c|c|c|c|c|}
\hline Variables & $\mathrm{V} 0(n=55)$ & $\mathrm{V} 1(n=53)$ & $\mathrm{V} 2(n=51)$ & $\Delta \mathrm{V} 1(n=52)$ & $\Delta \mathrm{V} 2(n=51)$ \\
\hline ENMO (mg/day) & $20.4(7.7)$ & $21.9(7.5)$ & $21.8(7.4)$ & $1.6(4.6)$ & $1(3.9)$ \\
\hline Physician global activity $(0-10)$ & $2.7(2.2)$ & $0.9(1.3)^{\# \#}$ & $0.8(1.2)^{\# \#}$ & $-17.7(22.5)$ & $-18.4(27.8)$ \\
\hline Patient global activity $(0-10)$ & $4.6(2.2)$ & $3.4[2]^{* *}$ & $2.8[2]^{* *}$ & $-11.9(25.2)$ & $-17.3(25.5)$ \\
\hline MMT8 total score $(0-150)$ & $135.4(19.9)$ & $138.3(20.2)^{\#}$ & $138.7(20.2)$ & $2.1(5.6)$ & $2.3(6.2)$ \\
\hline AST level (IU/L) & $76.9(104.2)$ & $31.9(14.2)^{\# \#}$ & $30[16] \# \#$ & $-53.2(117.7)$ & $-48.6(110.8)$ \\
\hline ALT level (IU/L) & $67(93.4)$ & $32.1(23.3) \# \#$ & $33.3[33]^{\# \#}$ & $-38.3(88.7)$ & $-33.4(80.9)$ \\
\hline LDH level (IU/L) & $662.2(442.8)$ & $482.2(139.9) \# \#$ & $410.7(138) \# \#$ & $-21(46)$ & $-52.9(86.8)$ \\
\hline Extra-muscular global activity $(0-10)$ & $1.2(1.4)$ & $0.7[1]]^{\# \#}$ & $0.4(0.7)^{\# \#}$ & $-5.2(11.5)$ & $-6.3(11.3)$ \\
\hline Sum of absolute percentage & 1 & 1 & 1 & $22.8(27.3)$ & $23.7[31]$ \\
\hline Mingazzini (0-75 s) & $39.5(30.7)$ & $50.7(31.6) \# \#$ & $50.9(30.5)^{\# \#}$ & $14.3[30]$ & $16.7(28.3)$ \\
\hline \multicolumn{6}{|l|}{ Patient reported outcomes } \\
\hline SF-36-general health (\%) & $43.3[21]$ & $50.6(24.1)^{*}$ & $52.7[23]^{* *}$ & $7.7(17.8)$ & $9.1(16.2)$ \\
\hline SF-36-physical functioning (\%) & $48.1(29.6)$ & $57.9(31.4)^{*}$ & $64.7(28.9)^{* *}$ & $10.6(28.6)$ & $14.5(26.5)$ \\
\hline SF-36-social functioning (\%) & $57.4(31.3)$ & $66.8(27.8)^{*}$ & $71.2(27.7)^{* *}$ & $8.9(24.7)$ & $11.5(19.9)$ \\
\hline SF-36- physical role (\%) & $44.9(30.5)$ & $62.6(32.2)^{* *}$ & $65.1(27.8)$ & $17.4(27.7)$ & $17.6(30.1)$ \\
\hline SF-36- emotional role (\%) & $56.3(32.1)$ & $72.4(30.2)^{* *}$ & $77(22.9)^{* *}$ & $15.1(28.8)$ & $17.9(26.4)$ \\
\hline SF-36-emotional wellbeing (\%) & $57.3[19]$ & $66.3(18.8)^{* *}$ & $69.7(15.6)^{* *}$ & $9.4[16]$ & $10.7(14.9)$ \\
\hline SF-36-energy/fatigue (\%) & $42(19.8)$ & $54.4[22]^{* *}$ & $55.1(20.8)^{* *}$ & $12.4(21.8)$ & $10.5(18.9)$ \\
\hline SF-36-pain (\%) & $64.9(25.6)$ & $75.7(21.4)$ & $73.2(23.2)$ & $10.2(22.2)$ & $6.1(26.1)$ \\
\hline HAD-depression & $5.6(3.6)$ & $4.4(3.8)^{*}$ & $4.6(3.7)^{*}$ & $-9.2(14.6)$ & $-6.1(11.8)$ \\
\hline HAD-anxiety & $8(3.8)$ & $6.4(3.4)^{*}$ & $5.8(3.5)^{* *}$ & $-7(14.9)$ & $-8.8(15.5)$ \\
\hline Rituximab (\%) & 13 & 10 & 14 & -4 & 0 \\
\hline IVIg (\%) & 50 & 58 & 47 & 8 & -6 \\
\hline
\end{tabular}

ENMO, Euclidian norm minus one; HAQ Health Assessment Questionnaire; CK, creatine kinase; IVIg, intravenous immunoglobulin; MMT8, manual muscle testing 8; HHD, hand held dynamometry; HAD, Hospital Anxiety and Depression scale; SF-36, Short Form Health Survey; IVIg, intravenous immunoglobulin; Bonferroni's post-hoc tests were performed if repeated measures ANOVA demonstrated significant main effect of time on normally distributed variables $\left({ }^{* * *}, p<0.05, p<0.001\right.$, respectively); Bonferroni's post-hoc tests were performed if repeated Friedman test demonstrated significant main effect of time on non-normally distributed variables (\#\#\#, $p<0.05, p<0.001$, respectively). $\Delta$ V1 and $\Delta$ V2, changes expressed as absolute percent change ([final value - baseline value] / range $\times 100$ ) or absolute changes (see manuscript for details). Except for SF-36 variables,\% indicates proportion/prevalence.

47.7\% had unchanged status, $14.4 \%$ had minimal improvement, $22.2 \%$ had moderate improvement, and $11.1 \%$ had major improvement.

Agreement between the adapted ACR/EULAR response criteria and change in PA at follow-up is shown in Table 4. All patients with a worsened ACR/EULAR clinical status had unchanged PA. Unchanged ACR/EULAR clinical status was associated with unchanged PA. Level of agreement between ACR/EULAR status and changes in PA was associated with the degree of improvement, i.e. agreement was low (15\%) for minimal improvement, mild (45\%) for moderate improvement, and high (90\%) for major improvement. The Cohen's kappa was $0.32(p<0.001)$, which represents fair agreement according to Ashby et al. [25] classification.

We subsequently investigated differences in patients with moderate ACR/EULAR improvement with unchanged or increased PA. At follow-up, patients with unchanged PA had lower ACR/EULAR total score $(43.9 \pm 3.0 \%$ vs. $50.0 \pm 6.0 \%, p<0.01, d=-1.28)$, smaller changes in psoas HHD strength $(9.7 \pm 9.0 \%$ vs. $21.1 \pm 6.4 \%, p<0.01, d=-1.46)$, smaller changes in muscle enzymes (ALT: $-12 \pm 22 \%$ vs. $95 \pm 87 \%$, $p<0.05, d=1.29$; AST: $-14 \pm 28 \%$ vs. $-122 \pm 125 \%, p<0.05, d=1.18$; LDH: $5 \pm 19 \%$ vs. $-133 \pm 106 \%, p<0.05, d=1.81$ ), smaller changes in HAD depression score $(-4 \pm 8 \%$ vs. $-15 \pm 10 \%, p<0.05, d=1.26)$, and smaller changes in SF-36-energy/fatigue scores ( $12 \pm 12 \%$ vs. $32 \pm 23 \%$, $p<0.05, d=-1.09)$. Interestingly, no significant difference was found for change in the extra-muscular disease activity ( $-10 \pm 10$ vs. $-4 \pm 7$, $p=0.19$ ). Patients with unchanged PA at follow-up presented at inclusion a lower ENMO Z-score $(-1.3 \pm 0.0$ vs. $-0.66 \pm 0.75, p<0.05$, $d=-1.21$ ), lower percentage predicted psoas strength ( $50 \pm 5 \%$ vs. $63 \pm$ $11 \%, p<0.05, d=-1.58$ ), lower ALT/AST/LDH levels (all $p<0.05$ ), longer disease duration $(5.0 \pm 1.7$ years vs. $1.9 \pm 1.9$ years, $p<0.05, d=1.74$ ), poorer SF-36-emotional wellbeing and energy scores (all $p<0.05$ ), higher extra-muscular disease activity $(2.8 \pm 1.1$ vs. $1.1 \pm 1.1, p<0.05$, $d=1.57$ ), and lower patient-reported global disease activity (3.6 \pm 1.3 vs. $6.4 \pm 3.0, p<0.05, d=-1.21)$.

\section{Discussion}

Our study demonstrates that most patients with IIM display low PA compared to age-matched healthy individuals in the general population, highlighting the burden of IIM on patients' daily life. Muscle strength and patient reported physical function were major determinants of PA at inclusion and follow-up. However, PA change was also associated with change in depression and fatigue levels. At follow-up, all patients with worsened or unchanged clinical status 
A.

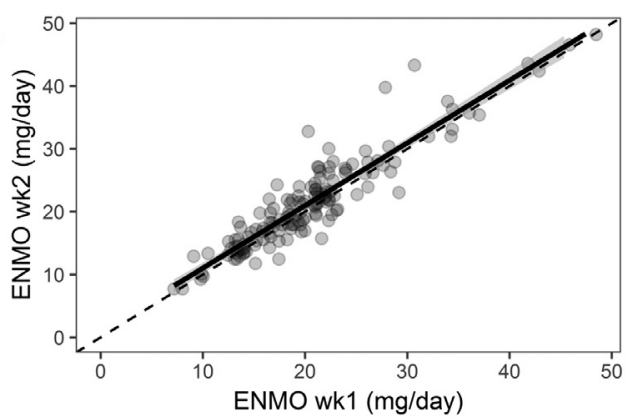

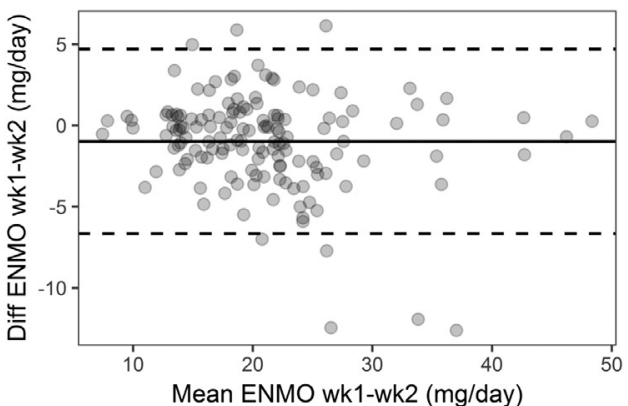

B.

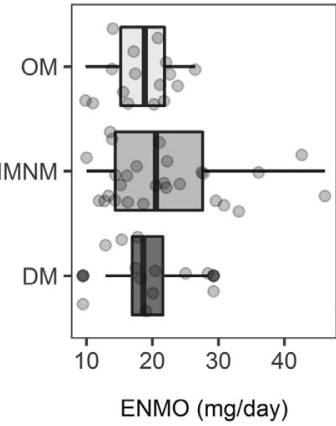

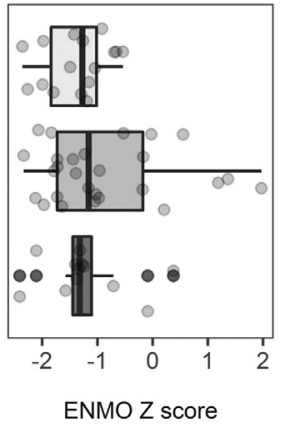
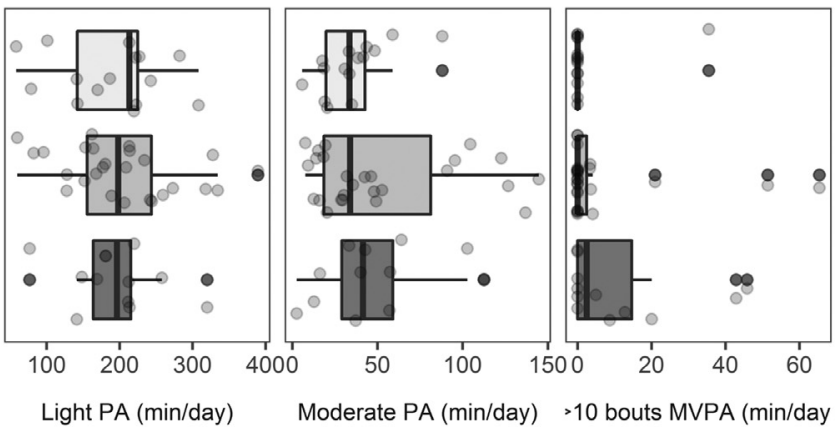

Fig. 1. Physical activity metrics at inclusion

Bland-Altman plot and regression analysis of Euclidian norm minus one (ENMO) during the first and second week of recordings (wk1 and wk2, respectively; Panel A). In the

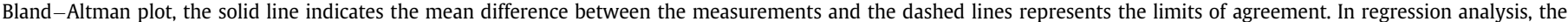
dashed line represents the identity line, and the solid line indicates the linear regression line with shaded 95\% confidence region. Physical activity metrics at baseline (Panel B).

had unchanged PA. Most patients (90\%) with a major improvement (as defined by the ACR/EULAR response criteria) had a significant increase in PA, whereas PA increase was observed in only half of the patients with a moderate improvement. Few patients with a minimal improvement displayed a significant increase in PA.
Our data demonstrate that monitoring PA using wrist-worn accelerometers is feasible in patients with IIM (nearly negligible non-wear time) [26-28] and display excellent absolute and relative reliability as previously demonstrated in patients cohorts with other conditions $[20,29,30]$.

Table 3

Relationships between physical activity and other variables.

\begin{tabular}{|c|c|c|c|c|c|}
\hline \multicolumn{3}{|l|}{ At inclusion } & \multicolumn{3}{|l|}{ At follow-up } \\
\hline Variables & rho & p value & Changes in variables* & rho & p value \\
\hline Core set measures & & & Core set measures & & \\
\hline Physician global activity $(0-10)$ & -0.253 & 0.065 & Physician global activity & -0.403 & $<0.001$ \\
\hline Patient global activity $(0-10)$ & -0.235 & 0.087 & Patient global activity & -0.351 & $<0.001$ \\
\hline MMT8 total score $(0-150)$ & 0.424 & $<0.01$ & MMT8 total score & 0.435 & $<0.001$ \\
\hline HAQ & 0.52 & $<0.001$ & HAQ & -0.456 & $<0.001$ \\
\hline CK level (IU/L) & 0.024 & 0.867 & CK level & -0.426 & $<0.001$ \\
\hline AST level (IU/L) & -0.203 & 0.141 & AST level & -0.417 & $<0.001$ \\
\hline ALT level (IU/L) & -0.108 & 0.436 & ALT level & -0.362 & $<0.001$ \\
\hline LDH level (IU/L) & -0.088 & 0.566 & LDH level & -0.510 & $<0.001$ \\
\hline \multirow[t]{2}{*}{ Extra-muscular global activity $(0-10)$} & 0.148 & 0.287 & Extra-muscular global activity & -0.221 & $<0.05$ \\
\hline & & & Sum of absolute percentage & 0.540 & $<0.001$ \\
\hline Muscle assessment & & & Muscle assessment & & \\
\hline Deltoid HDD (\% pred) & 0.320 & $<0.05$ & Deltoid HDD & 0.162 & 0.128 \\
\hline Psoas HDD (\% pred) & 0.292 & $<0.05$ & Psoas HDD & 0.314 & $<0.01$ \\
\hline Barré (sec) & 0.349 & $<0.01$ & Barré & 0.268 & $<0.01$ \\
\hline Mingazzini (sec) & 0.286 & $<0.05$ & Mingazzini & 0.344 & $<0.001$ \\
\hline Patient reported outcomes & & & Patient reported outcomes & & \\
\hline SF-36 general health (\%) & 0.157 & 0.256 & SF-36 general health & 0.123 & 0.215 \\
\hline SF-36 physical functioning (\%) & 0.384 & $<0.01$ & SF-36 physical functioning & 0.423 & $<0.001$ \\
\hline SF-36 physical role (\%) & 0.148 & 0.286 & SF-36 physical role & 0.158 & 0.111 \\
\hline SF-36 emotional role (\%) & 0.144 & 0.299 & SF-36 emotional role & 0.238 & $<0.05$ \\
\hline SF-36 emotional wellbeing (\%) & -0.010 & 0.945 & SF-36 emotional wellbeing & & \\
\hline SF-36-energy/fatigue (\%) & 0.210 & 0.127 & SF-36-energy/fatigue & 0.440 & $<0.001$ \\
\hline SF-36-pain (\%) & 0.333 & $<0.05$ & SF-36-pain & 0.214 & $<0.05$ \\
\hline HAD-depression & -0.179 & 0.312 & HAD-depression & -0.460 & $<0.001$ \\
\hline HAD-anxiety & -0.005 & 0.978 & HAD-anxiety & & \\
\hline
\end{tabular}

Dependent variable was ENMO Z score at inclusion and changes in ENMO at follow-up; MMT8, manual muscle testing 8; HAQ Health Assessment Questionnaire; CK, creatine kinase; AST, aspartate transaminase; ALT, alanine transaminase; LDH, lactate dehydrogenase; HHD, hand held dynamometry; SF-36, Short Form Health Survey; HAD, Hospital Anxiety and Depression scale. * changes expressed as absolute percent change ([final value - baseline value] / range $\times 100$ ) or absolute changes (see manuscript for details). 

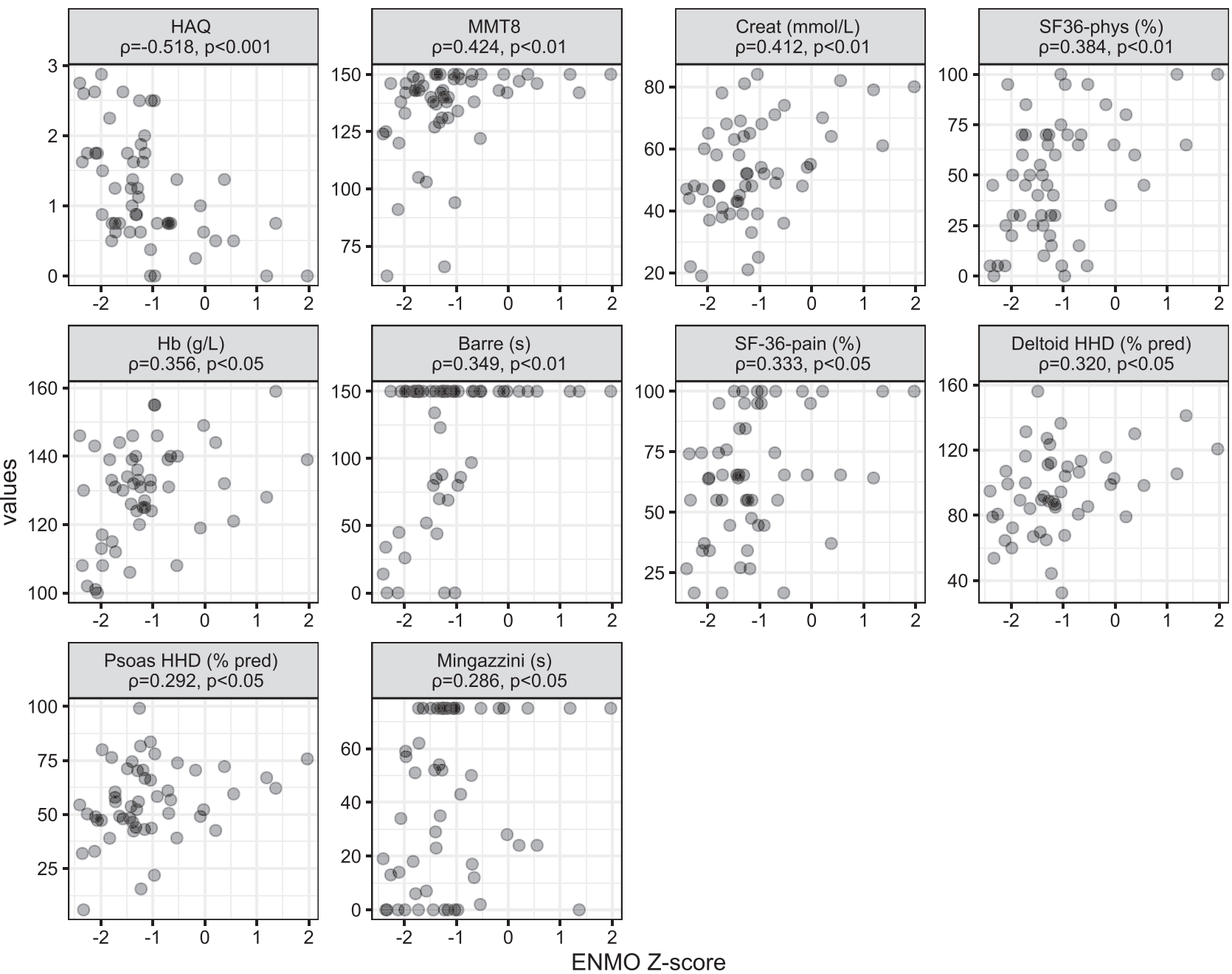

Fig. 2. Significant relationships between physical activity and other variables at inclusion

Significant correlations ordered from top left to lower right according to strength of association as computed using Spearman's correlation (p). HAQ Health Assessment Ques-

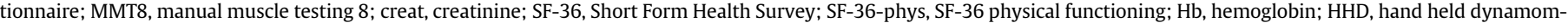
etry; ENMO Z-score, Euclidian norm minus one expressed as a Z-score.

PA was associated with objective muscle disease measures (MMT8, HHD, endurance, creatinine level) as well as patient-reported outcomes (general health as assessed by the HAQ functioning capacities and pain as assessed using the SF-36 questionnaire, see Fig. 2). Some variables (e.g. MMT8, Barré, Mingazzini) displayed ceiling and/ or floor effects in many patients. Consequently, and although nonparametric tests were used, correlations between PA and the latter variables must be interpreted with caution.

At follow-up, change in PA correlated with MMT8 total score and accordingly, with clinical status change (ACR/EULAR response criteria). This may be explained, at least in part, by the fact that the ACR/ EULAR response criteria include many of the variables correlated to $\mathrm{PA}$ at baseline. The agreement between change in PA and ACR/EULAR status was excellent when major improvement occurred suggesting that patients captured in this category correspond to patients with a clinically meaningful improvement (i.e. translating to significant change in PA). Yet, only a half of patients with a moderate improvement had an increase in PA, suggesting that this ACR/EULAR category captures a heterogeneous group of patients associated with and without significant change in PA. A potential explanation is that mild improvement in the MMT8 score may be overlooked as physicians have difficulty discriminating most Medical Research Council categories (5-points scale) especially for mild variations [31]. In addition, small increase in muscle strength does not necessary translate into substantial improvements in functional abilities [32]. We also stress that PA was monitored continuously for 2 weeks in real life suggesting that this measure may be more reliable to capture changes than a one point-in-time evaluation point at a medical visit. As only $10 \%$ of patients with minimal improvement displayed changes in PA, it suggests that this ACR/EULAR category defines a homogeneous group of patients with very low probability of presenting a significant change in PA. Altogether, these findings suggest that an increase in $\mathrm{PA}>4.10$ $\mathrm{mg}$ /day (i.e. defined as the least significant change) could serve as a useful, feasible, and objective tool to define a clinically relevant reallife outcome.

Predictors of mismatch between ACR/EULAR response criteria and change in PA were smaller change in muscle strength and muscle enzymes. Interestingly, our data also suggest that disease duration and long-term damage may prevent patients from displaying an improved PA, as longer disease duration and lower patient-reported global disease activity were predictors of unchanged PA. The extramuscular disease activity was not a predictor of mismatch in our study, suggesting that the absence of increase in PA in patients with ACR/EULAR moderate improvement is not explained by active extramuscular features. We acknowledge that we were unable to fully address the importance of each extra-muscular features individually and their possible impact on PA behavior (pain related to arthritis, shortness of breath related to lung involvement). Another limitation 
$\triangle E N M O$ Reduced Unchanged Increased timepoint $\bigcirc \mathrm{V} 1 \Delta \mathrm{V} 2$
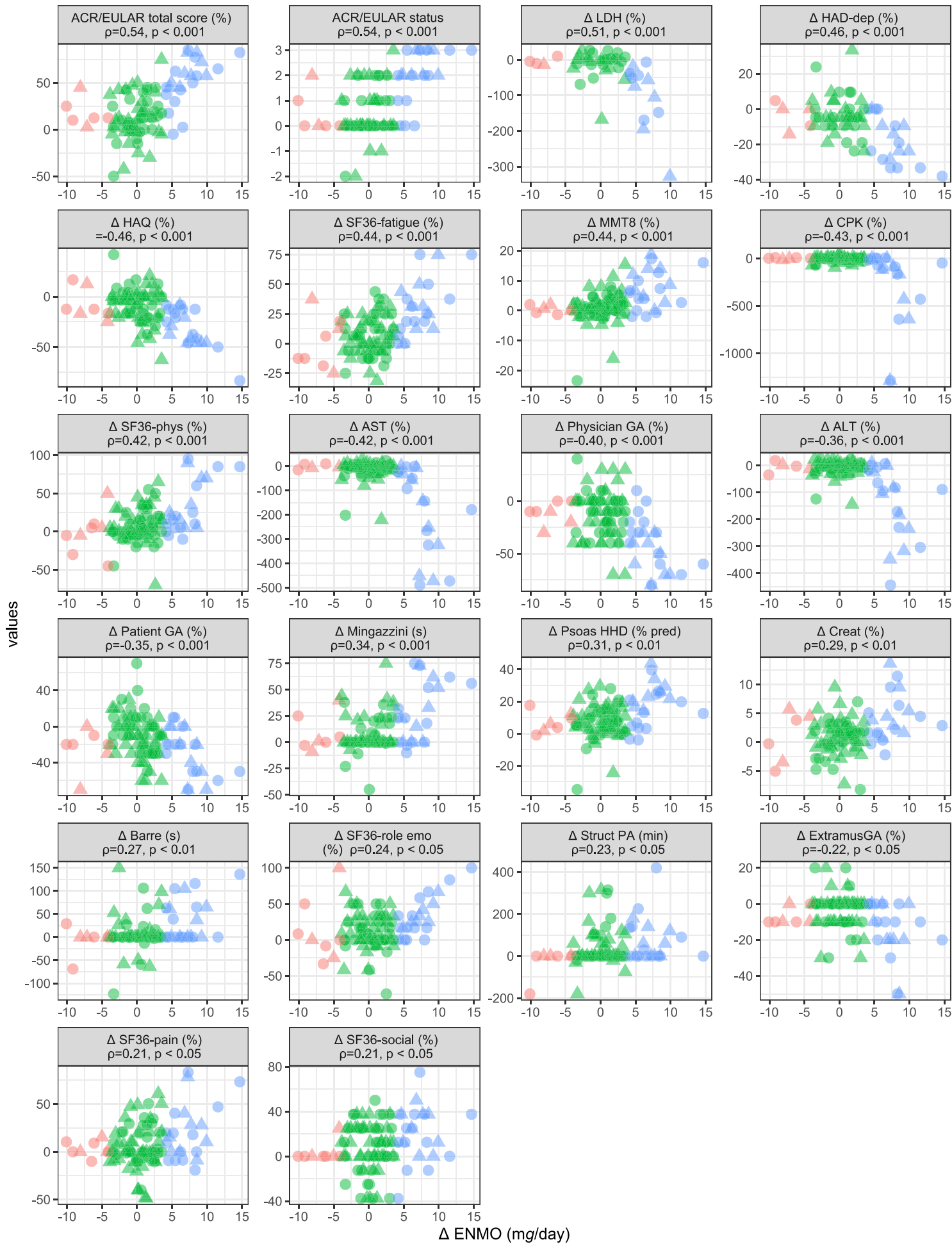

Fig. 3. Significant relationships between changes in physical activity and changes in other variables at follow-up

The $\mathrm{x}$-axis represents the absolute change in Euclidian norm minus one (ENMO). The y-axis represents changes in variables ( $\Delta$ ). Shapes indicate first and second follow-up visits

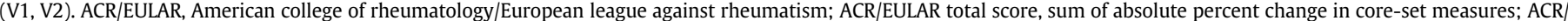

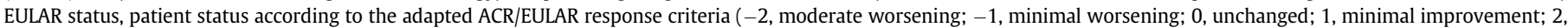

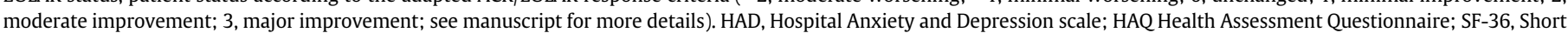

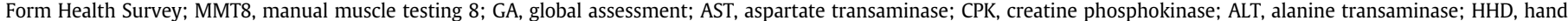
held dynamometry; creat, creatinine; Struct PA, structured physical activity; ExtramusGA, extra-muscular global assessment. 
Table 4

Table of contingency of agreement between adapted ACR/EULAR response criteria and change in PA at follow-up

\begin{tabular}{lllll}
\hline \multirow{2}{*}{ Adapted ACR/EULAR status* } & \multicolumn{2}{l}{ Change in ENMO } & & \\
& decreased & unchanged & increased & \% match \\
\hline Moderate worsening & 0 & 2 & 0 & 0 \\
Minimal worsening & 0 & 2 & 0 & 0 \\
Unchanged & 6 & 34 & 3 & 79 \\
Minimal improvement & 1 & 10 & 2 & 15 \\
Moderate improvement & 1 & 10 & 9 & 45 \\
Major improvement & 0 & 1 & 9 & 90 \\
\hline
\end{tabular}

ACR/EULAR, American college of rheumatology/European league against rheumatism. Definition of worsening clinical status were also computed by adapting the original definitions as follow: $-40 \leq$ minimal worsening $\leq-20 ;-60 \leq$ moderate worsening $\leq-40$; $\geq 60$ major worsening. gray boxes indicate expected match.

is that we cannot exclude the impact of active DM skin rash on PA behavior as cutaneous disease activity is associated with poorer quality of life [33].

Another important point is the negative impact of wellbeing and emotional variables on PA behaviors suggested by smaller changes in depression score and fatigue level that were also predictors of mismatch between ACR/EULAR response criteria and change in PA. PA is not only influenced by physical/physiological factors but also by behavioral, demographic, and socioeconomic factors [34,35]. Our data show that change in HAD depression score was correlated to change in PA in accordance with previous data demonstrating the association between PA and mental health [30,31]. This observation highlights the importance of considering mood/psychological disturbances when patients with IIM present with lower than expected PA level when disease activity is improving. Psychotherapeutic interventions such as patient-centered cognitive behavioral therapy may contribute to promote more active lifestyle and thus, improve outcomes and reduce comorbidities associated with poor mental health and physical inactivity. Socioeconomic events (e.g. relocation, change in social environment) are very likely to affect PA and should be recorded in such studies. PA assessment must be highly contextualized and not considered as a unique tool for disease activity monitoring, especially for patients with predominant extra-muscular involvement. We also acknowledge that most of our patients were recruited in a stable state and that these acquisitions remain to be evaluated in a clinical trial.

To conclude, this study demonstrated that significant increase in PA occurs in most patients with major improvement (as defined by ACR/EULAR IIM response criteria), in only half of the patients with moderate improvement, and almost never in patients with mild improvement. We also identified that significant increase in PA in patients with clinical improvement is less likely to occur in the absence of improvement in psychological status.

Altogether, our data support objective assessment of PA in future prospective clinical trials and clinical practice to improve the assessment of intervention and clinical management of patient with IIM.

\section{Author contribution}

Conceptualization and design: OL-C, DB, OB, JYH and YA

Acquisition of data: OL-C, DB, PGJ, MV, NC, BH, AR, OB, JYH and YA

Analysis and interpretation: OL-C, DB, RA, OB, JYH and YA

Critical revision of the manuscript for important intellectual con-

tent: OL-C, DB, PGJ, MV, NC, BH, AR, RA, OB, JYH and YA

OL-C, DB, and YA drafted the original version of the manuscript.

Final approval of the manuscript: all authors

\section{Funding}

This research was supported by the Association Française Contre les Myopathies(AFM).

\section{Disclosure}

Dr. Landon-Cardinal is the recipient of Clinical Fellowship awards from the Université de Montréal Rheumatology Program - Abbvie Educational Grant and the Association des médecins rhumatologues du Québec - Visithan-Khy Educational Grant. DB, PGJ, MV, NC, BH, AR, RA, OB, JYH and YA report no disclosure.

\section{Acknowledgement}

We gratefully thank all the volunteers who participated in this study. We also thank Dr Vincent T. van Hees and other contributors for maintaining the open-source GGIR R-package. We sincerely acknowledge Dr. Simone Birnbaum for English editing.

\section{References}

[1] Leclair V, Regardt M, Wojcik S, Hudson M. Canadian Inflammatory Myopathy Study (CIMS). In: Kuwana M, editor. editor, 2016 Aug 9, editor. Health-Related quality of life (HROoL) in idiopathic inflammatory myopathy: a systematic review, 11. PLOS ONE; 2016 Aug 9:e0160753.

[2] Benveniste O, Stenzel W, Allenbach Y. Advances in serological diagnostics of inflammatory myopathies. Curr Opin Neurol 2016 Oct;29(5):662-73.

[3] Rider LG, Giannini EH, Brunner HI, Ruperto N, James-Newton L, Reed AM, et al International consensus on preliminary definitions of improvement in adult and juvenile myositis. Arthritis Rheumatism 2004 Jul;50(7):2281-90.

[4] Aggarwal R, Rider LG, Ruperto N, Bayat N, Erman B, Feldman BM, et al. 2016 American College of Rheumatology/European League Against Rheumatism Criteria for Minimal, Moderate, and Major Clinical Response in Adult Dermatomyositis and Polymyositis: an International Myositis Assessment and Clinical Studies Group/Paediatric Rheu: ACR/EULAR CRITERIA FOR CLINICAL RESPONSE IN ADULT DERMATOMYOSITIS AND POLYMYOSITIS. Arthritis Rheumatol 2017 May;69 (5):898-910.

[5] van Hees VT, Fang Z, Langford J, Assah F, Mohammad A, da Silva ICM, et al. Autocalibration of accelerometer data for free-living physical activity assessment using local gravity and temperature: an evaluation on four continents. J Appl Physiol 2014 Oct 1;117(7):738-44.

[6] van Hees VT, Gorzelniak L, Dean León EC, Eder M, Pias M, Taherian S, et al. Separating movement and gravity components in an acceleration signal and implications for the assessment of human daily physical activity. PLoS ONE 2013;8(4): e61691.

[7] Bachasson D, Landon-Cardinal O, Benveniste O, Hogrel J-Y, Allenbach Y. Physical activity monitoring: a promising outcome measure in idiopathic inflammatory myopathies. Neurology 2017 Jul 4;89(1):101-3.

[8] Oldroyd A, Little MA, Dixon W, Chinoy H. A review of accelerometer-derived physical activity in the idiopathic inflammatory myopathies. BMC Rheumatology 2019 Oct $21 ; 3(1): 41$

[9] Lundberg IE, Tjärnlund A, Bottai M, Werth VP, Pilkington C, Visser M de, et al 2017 European League Against Rheumatism/American College of Rheumatology classification criteria for adult and juvenile idiopathic inflammatory myopathies and their major subgroups. Ann Rheum Dis. 2017;76(12):1955-64.

[10] Allenbach Y, Mammen AL, Benveniste O, Stenzel W, Allenbach Y, Amato A, et al 224th ENMC International Workshop. Neuromuscular Disorders 2018 Jan;28 (1):87-99.

[11] Mariampillai K, Granger B, Amelin D, Guiguet M, Hachulla E, Maurier F, et al. Development of a New Classification System for Idiopathic Inflammatory Myopathies Based on Clinical Manifestations and Myositis-Specific Autoantibodies. JAMA Neurol 2018 Dec 1;75(12):1528-37.

[12] Troyanov Y, Targoff IN, Tremblay J-L, Goulet J-R, Raymond Y, Senécal J-L. Nove classification of idiopathic inflammatory myopathies based on overlap syndrome features and autoantibodies: analysis of 100 French Canadian patients. Medicine (Baltimore) 2005 Jul:84(4):231-49.

[13] Lloyd TE, Mammen AL, Amato AA, Weiss MD, Needham M, Greenberg SA. Evaluation and construction of diagnostic criteria for inclusion body myositis. Neurology 2014 Jul 29;83(5):426-33.

[14] Benveniste O, Guiguet M, Freebody J, Dubourg O, Squier W, Maisonobe T, et al. Long-term observational study of sporadic inclusion body myositis. Brain 2011 Nov 1;134(11):3176-84.

[15] Rider LG, Koziol D, Giannini EH, Jain MS, Smith MR, Whitney-Mahoney K, et al Validation of manual muscle testing and a subset of eight muscles for adult and juvenile idiopathic inflammatory myopathies. Arthritis Care Res (Hoboken) 2010 Apr;62(4):465-72.

[16] Harbo T, Brincks J, Andersen H. Maximal isokinetic and isometric muscle strength of major muscle groups related to age, body mass, height, and sex in 178 healthy subjects. Eur J Appl Physiol 2012 Jan;112(1):267-75.

[17] Hogrel J-Y, Payan CA, Ollivier G, Tanant V, Attarian S, Couillandre A, et al. Development of a French isometric strength normative database for adults using quantitative muscle testing. Arch Phys Med Rehabil 2007 Oct;88(10):1289-97.

[18] Landon-Cardinal O, Devilliers H, Chavarot N, Mariampillai K, Rigolet A, Hervier B, et al. Responsiveness to Change of 5-point MRC scale, Endurance and Functional 
Evaluation for Assessing Myositis in Daily Clinical Practice. J Neuromuscul Dis. 2019;6(1):99-107.

[19] White T, Westgate K, Wareham NJ, Brage S. Estimation of Physical Activity Energy Expenditure during Free-Living from Wrist Accelerometry in UK Adults. PLoS ONE 2016;11(12):e0167472.

[20] Wolff-Hughes DL, McClain JJ, Dodd KW, Berrigan D, Troiano RP. Number of accelerometer monitoring days needed for stable group-level estimates of activity. Physiol Meas 2016;37(9):1447-55.

[21] Esliger DW, Rowlands AV, Hurst TL, Catt M, Murray P, Eston RG. Validation of the GENEA Accelerometer. Med Sci Sports Exerc 2011 Jun;43(6):1085-93.

[22] Doherty A, Jackson D, Hammerla N, Plötz T, Olivier P, Granat MH, et al. In: Buchowski M, editor. editor. Large scale population assessment of physical activity using wrist worn accelerometers: the uk biobank study, 12. PLOS ONE; 2017 Feb 1:e0169649.

[23] Aaronson NK, Acquadro C, Alonso J, Apolone G, Bucquet D, Bullinger M, et al International Quality of Life Assessment (IQOLA) Project. Qual Life Res 1992 Oct; 1 (5):349-51.

[24] Zigmond AS, Snaith RP. The hospital anxiety and depression scale. Acta Psychiatr Scand 1983 Jun;67(6):361-70.

[25] Ashby D.Practical statistics for medical research. Douglas G. Altman, Chapman and Hall, London, 1991. No. of pages: 611. Price: £32.00. Statistics in Medicine. 1991;10(10):1635-6.

[26] Duncan MJ, Brown WJ, Burrows TL, Collins CE, Fenton S, Glozier N, et al. Examining the efficacy of a multicomponent m-Health physical activity, diet and sleep intervention for weight loss in overweight and obese adults: randomised controlled trial protocol. BMJ Open 2018 Oct 30;8(10):e026179.

[27] Shelley J, Fairclough SJ, Knowles ZR, Southern KW, McCormack P, Dawson EA, et al. A formative study exploring perceptions of physical activity and physical activity monitoring among children and young people with cystic fibrosis and health care professionals. BMC Pediatr 2018 Oct 23:18(1):335.
[28] Cassidy S, Fuller H, Chau J, Catt M, Bauman A, Trenell MI. Accelerometer-derived physical activity in those with cardio-metabolic disease compared to healthy adults: a UK Biobank study of 52,556 participants. Acta Diabetol 2018 Sep;55 (9):975-9.

[29] Skender S, Schrotz-King P, Böhm J, Abbenhardt C, Gigic B, Chang-Claude J, et al. Repeat physical activity measurement by accelerometry among colorectal cancer patients-feasibility and minimal number of days of monitoring. BMC Res Notes 2015 Jun 6;8:222.

[30] Bassett DR. Validity and reliability issues in objective monitoring of physical activity. Res Q Exerc Sport 2000 Jun;71(2 Suppl):S30-6.

[31] Vanhoutte EK, Faber CG, van Nes SI, Jacobs BC, van Doorn PA, van Koningsveld R, et al. Modifying the Medical Research Council grading system through Rasch analyses. Brain 2012 May; 135(5):1639-49.

[32] Hogrel J-Y, Allenbach Y, Canal A, Leroux G, Ollivier G, Mariampillai K, et al. Fouryear longitudinal study of clinical and functional endpoints in sporadic inclusion body myositis: implications for therapeutic trials. Neuromuscul Disord 2014 Jul:24(7):604-10.

[33] Robinson ES, Feng R, Okawa J, Werth VP. Improvement in the cutaneous disease activity of patients with dermatomyositis is associated with a better quality of life. British Journal of Dermatology 2015 Jan;172(1):169-74.

[34] Achttien R, van Lieshout J, Wensing M, van der Sanden MN, Staal JB. Symptoms of depression are associated with physical inactivity but not modified by gender or the presence of a cardiovascular disease; a cross-sectional study. BMC Cardiovasc Disord 2019 Apr 25;19(1):95.

[35] Sabia S, van Hees VT, Shipley MJ, Trenell MI, Hagger-Johnson G, Elbaz A, et al. Association Between Questionnaire- and Accelerometer-Assessed Physical Activity: the Role of Sociodemographic Factors. Am. J. Epidemiol. 2014 Mar 15;179 (6):781-90. 